\title{
An Application of ASP Theories of Intentions to Understanding Restaurant Scenarios: Insights and Narrative Corpus
}

\author{
QINGLIN ZHANG
}

CHRIS BENTON

DANIELA INCLEZAN

Miami University, College of Engineering 83 Computing, Oxford OH 45056, USA

(e-mail: zhangq7, bentoncl, inclezd@miamioh.edu)

submitted 1 January 2003; revised 1 January 2003; accepted 1 January 2003

\begin{abstract}
This paper presents a practical application of Answer Set Programming to the understanding of narratives about restaurants. While this task was investigated in depth by Erik Mueller, exceptional scenarios remained a serious challenge for his script-based story comprehension system. We present a methodology that remedies this issue by modeling characters in a restaurant episode as intentional agents. We focus especially on the refinement of certain components of this methodology in order to increase coverage and performance. We present a restaurant story corpus that we created to design and evaluate our methodology. Under consideration in Theory and Practice of Logic Programming (TPLP).
\end{abstract}

KEYWORDS: Answer Set Programming, natural language understanding, intentional agents

\section{Introduction}

In this paper, we present an application of Answer Set Programming (ASP) to the understanding of restaurant narratives. Dining at a restaurant is a stereotypical human activity, i.e., a sequence of actions normally performed in a certain order by one or more actors, according to cultural conventions. Automating a deep understanding of stories about stereotypical human activities is a more difficult task than that of understanding other types of narratives because a larger number of events that are part of the activity are not explicitly mentioned in the text, with the assumption that readers will be able to fill in the gaps based on their shared cultural knowledge. Consider the following example:

Example 1 (Normal scenario from (Mueller 2007))

Nicole went to a vegetarian restaurant. She ordered lentil soup. The waitress set the soup in the middle of the table. Nicole enjoyed the soup. She left the restaurant.

The story in Example 1 does not mention that the waitress went to the kitchen to get the soup nor that Nicole paid for her meal, as these actions are implicitly assumed.

We chose to focus specifically on restaurants with table service because stories about 
this domain involve more actors, performing more actions, and interacting in more complex ways than in other types of restaurants (e.g., fast food restaurants). As a consequence, knowledge representation and reasoning techniques become relevant to understanding stories about the chosen stereotypical activity, while an automated learning of the sequence of events that form this activity would be very difficult, as indicated in Section 2. Our work is applicable to stories about other stereotypical activities.

Schank and Abelson (1977) proposed modeling stereotypical human activities as scripts, i.e., "standardized sequences of events" (Barr and Feigenbaum 1981), and Mueller conducted substantial research in this direction (e.g., (Mueller 2004)), including work on the restaurant domain specifically (Mueller 2007). However, his system was not able to understand stories describing exceptional scenarios like the ones in Examples 2 and 3 below because of the rigid structure of scripts - actions in the script are assumed to always occur in the exact order specified in the script.

Example 2 (Serendipity)

Nicole went to a vegetarian restaurant. She ordered lentil soup. When the waitress brought her the soup, she told Nicole that it was on the house. Nicole enjoyed the soup and then left. (The reader should understand that Nicole did not pay for the soup.)

Example 3 (Diagnosis)

Nicole went to a vegetarian restaurant. She ordered lentil soup. The waitress brought her a miso soup instead. (The reader is supposed to produce some explanations for what may have gone wrong: either the waitress or the cook misunderstood the order.)

We have argued (Zhang and Inclezan 2017 Inclezan et al. 2018) that modeling actors in a restaurant scenario as goal-driven intentional agents is needed in order to be able to process exceptional restaurant scenarios in addition to normal ones. We have proposed to use theories of intentions written in ASP (Baral and Gelfond 2005) or easily translatable into ASP (Blount 2013 Blount et al. 2015) to model the characters in a restaurant episode as intentional agents, and concluded that our methodology has a wider coverage than script-based approaches.

In this paper, we investigate remaining research questions related to the proposed methodology and present a corpus of restaurant stories that we built in order to evaluate our methodology, and which we make publicly available.

Our first research question studies the impact in terms of coverage and performance of modeling all characters in a restaurant scenario as goal-driven intentional agents as defined by Blount et al. (2015) versus viewing only the main character, the customer, as such, and modeling other characters using a simpler theory of intentions by Baral and Gelfond (2005). The second research question investigates the optimal structure for the representation of the stereotypical activity of dining at a restaurant from the point of view of each character. We envision the restaurant corpus that we constructed, restaurant-1.0, as a resource to be used in future research on stereotypical activities, but also a useful benchmark for the NLP, natural language understanding, KRR, and ASP communities.

The contributions of this work are as follows:

- We demonstrate that, by using ASP theories of intentions, our proposed methodology can reason about exceptional scenarios that can not be processed using traditional, 
script-based approaches. Thus, we introduce and highlight an important application area for the ASP body of work on theories of intentions.

- We indicate that modeling only the customer role as a goal-driven intentional agent presents advantages in terms of performance, while having only a moderate negative impact on coverage.

- We provide guidelines for structuring the representation of stereotypical human activities based on lessons learned from serendipitous scenarios like the one in Example 2, which require a hierarchical structure where activities have sub-activities with sub-goals, and scenarios involving diagnosis like the one in Example 3 , which require paying attention to the parameters of the activity.

- We introduce and make available a corpus of restaurant stories accompanied by their ASP logic forms.

In what follows, we start by discussing related work and then describe the proposed methodology for reasoning about restaurant stories. Next, we explore the two research questions connected to our methodology. We then briefly present the application of our refined methodology on a few illustrative stories. We present the restaurant story corpus that we created and end with conclusions and future work.

\section{Related Work}

Restaurant Narratives. Erik Mueller's work is based on the hypothesis that readers of a text understand it by constructing a mental model (Craik 1943) of the narrative (Johnson-Laird 1983 van Dijk and Kintsch 1983). Mueller's system (2007) showed a deep understanding of restaurant narratives by answering questions about time and space aspects that were not necessarily mentioned explicitly in the text. His system relied on two important pieces of background knowledge: a commonsense knowledge base about actions occurring in a restaurant, their effects and preconditions, encoded in Event Calculus (Shanahan 1997); and a script describing a sequence of actions performed in a normal unfolding of a restaurant episode. The script was much more detailed than those used in other systems, for instance Ng and Mooney's plan recognition software ACCEL (1992), and thus was able to demonstrate a more in-depth understanding.

The system processed English text using information extraction techniques in order to fill out slot values in a template. Table 1 shows the template constructed for the scenario in Example1. Note that the slot SCRIPT: LAST EVENT is filled with the value LEAVE, which corresponds to the customer's last action in the restaurant script. Next, the template was translated into a reasoning problem that contained: facts about the entities identified in the template; facts about the consecutive occurrence of all actions in the script up to the one corresponding to the value of the SCRIPT: LAST EVENT slot; and default information about the layout of the restaurant and the locations of different objects and characters. Then, the reasoning problem was expanded with the information in the commonsense knowledge base to compute models of the input restaurant scenario. Finally, questions about time and space aspects were automatically generated and answers were obtained from the model resulting in the previous step. Mueller's system was tested on 124 excerpts of texts retrieved from the web or Project Gutenberg collection, and answered correctly $70 \%$ of the test questions.

In terms of limitations of the system, the author acknowledged the lack of flexibility 
Table 1. Slot values for the template constructed for Example 1

\begin{tabular}{ll}
\hline \hline Slot & Slot Value \\
\hline SCRIPT: TYPE & ReSTAURANT \\
SCRIPT: LAST EVENT & LEAVE \\
RESTAURANT & "the vegetarian restaurant" \\
WAITER & "waitress" \\
CUSTOMER & "Nicole" \\
FOOD & "lentil soup" : "Nicole" \\
\hline \hline
\end{tabular}

of scripts, which resulted in scenarios with exceptional cases (or variations, such as an additional wine tasting step) not being processed correctly. For instance, for the scenario in Example 2, Mueller's system would detect LEAVE as the SCRIPT: LAST EVENT and thus construct the same template as the one for Example 1. As a result, the reasoning problem built from the template would include the fact that Nicole paid for the soup, since this action precedes the customer's action of leaving in the script, when in fact a human reader would infer that she did not pay because the soup was on the house. In the script-based approach, such a serendipitous scenario can only be solved by introducing a new script that does not contain the pay action. This means that the knowledge engineer would have to predict all possible exceptional scenarios and create a new script for each of them in advance.

Narrative Corpora. Mueller's two restaurant story corpora (2007), one based on Internet stories and the other on Project Gutenberg texts, are proprietary and thus unavailable. Reconstructing these corpora is a laborious task. General story corpora exist, but they do not apply to the subject of this research. For instance, the InScript narrative corpus (Modi et al. 2017) covers other stereotypical human activities (e.g. grocery shopping, taking the bus), but not the topic of dining in a restaurant with table service. The OMCS (Open Mind Common Sense) (Singh et al. 2002) and OMICS (Open Mind Indoor Common Sense) (Gupta and Kochenderfer 2004) corpora cited in earlier papers, though publicly available in the past, are no longer readily available and do not contain restaurant stories. The SMILE corpus (Regneri et al. 2010) contains stories about eating at a fast food restaurant, but not at an elegant restaurant. Gordon et al. (2007) processed and annotated an existing corpus of stories extracted from Internet web blogs. However, due to a complex agreement system for using the original web blog corpus, the data is not readily available to the public.

Automated Learning of Activities. In recent years, there has been an increased interest in automatically learning the sequence of events that forms a stereotypical activity (Chambers and Jurafsky 2008, Manshadi et al. 2008, Regneri et al. 2010). However, these approaches are only able to produce flat sequences of actions that are not associated with goals. Smith and Arnold (2009) are able to produce hierarchical plans, but these are not associated with goals either. As stated in the introduction, a hierarchical structure with sub-sequences and associated (sub-)goals is required for a system to be able to reason about exceptional scenarios like the ones in Examples 2 and 3 . Additionally, the targeted 
stereotypical activities in this unsupervised learning body of work do not include dining at a restaurant with table service and generally have only one actor (e.g., make coffee).

Activity Recognition. The task of automating the understanding of restaurant narratives is somewhat connected to activity recognition, in that it requires observing agents and their environment in order to complete the picture about the agents' actions and activities. However, unlike activity recognition, understanding restaurant narratives does not require identifying an agent's goal, which is always the same in our case (e.g., a customer entering a restaurant always seeks to become satiated). Gabaldon (2009) performed activity recognition using Baral and Gelfond's theory of intentions (2005) that did not consider goal-driven agents.

Preliminary Work. In an earlier version of this paper, Zhang and Inclezan (2017) presented an initial solution to the problem of reasoning about restaurant stories by using both of the existing theories of intentions. Inclezan et al. (2017; 2018) introduced the alternative approach of using the newer theory of intentions for modeling all characters in a restaurant story. Neither of these papers compare the two approaches nor include the work on the restaurant story corpus.

\section{Reasoning about Restaurant Stories in ASP}

As mentioned in the introduction, the script-based approach is not suitable for reasoning about exceptional scenarios because of the rigidity of scripts. In previous work (Zhang and Inclezan 2017; Inclezan et al. 2017; Inclezan et al. 2018), we proposed a new approach, capable of handling normal and exceptional scenarios, based on the idea of viewing the main character in a restaurant scenario (and possibly others) as a goal-driven intentional agent. We used theories of intentions to reason about the actions of such agents, coupled with a background knowledge base about actions and properties (fluents) relevant to the restaurant domain, as well as an encoding of the stereotypical activity itself, from the point of view of each character. In this section, we briefly introduce the two existing theories of intentions, written in ASP or languages closely related to ASP. We then outline our methodology and stress specifically the research questions that resulted from our preliminary work.

\subsection{Theory of Intended Actions by Baral and Gelfond}

Baral and Gelfond (2005) captured properties of intended actions in an ASP theory we denote by old $\mathcal{T} \mathcal{I}$ that had two main tenets: "Normally intended actions are executed the moment such execution becomes possible" (non-procrastination) and "Unfulfilled intentions persist" (persistence). Sequences of actions were modeled using predicates: sequence(s) ( $\mathbf{s}$ is a sequence); length $(\mathbf{n}, \mathbf{s})$ ( $\mathbf{n}$ is the length of sequence $\mathbf{s})$; and component $(\mathbf{s}, \mathbf{k}, \mathbf{x})$ (the

$\mathbf{k}^{t h}$ element of sequence $\mathbf{s}$ is $\mathbf{x}$, where $\mathbf{x}$ can be either an action or another sequence). An agent's intentions at different time points was captured by intend( $\mathbf{x}, \mathbf{i})$ (action/ sequence $\mathbf{x}$ is intended at time step $\mathbf{i}$ ). The theory was successfully used in activity recognition (Gabaldon 2009) and question answering about biological processes (Inclezan and Gelfond 2011), but was not sufficient for modeling goal-driven agents. We use the term simple

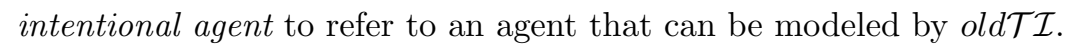




\subsection{Theory of Goal-Driven Intentional Agents by Blount et al.}

Blount and collaborators (Blount 2013: Blount et al. 2015) improved on the previous theory of intentions by considering goal-driven agents inspired by the Belief-Desire-Intention (BDI) model (Bratman 1987). For this purpose, each sequence of actions of an agent was associated with a goal that it was meant to achieve - the combination of the two was called an activity. Activities could have nested sub-activities, and were encoded using the predicates: activity $(\mathbf{m})(\mathbf{m}$ is an activity); $\operatorname{goal}(\mathbf{m}, \mathbf{g})$ (the goal of activity $\mathbf{m}$ is $\mathbf{g}$ ); length $(\mathbf{n}, \mathbf{m})$ (the length of activity $\mathbf{m}$ is $\mathbf{n}$ ); and component $\left(\mathbf{m}, \mathbf{k}, \mathbf{x}\right.$ ) (the $\mathbf{k}^{\text {th }}$ component of activity $\mathbf{m}$ is $\mathbf{x}$, where $\mathbf{x}$ is either an action or a sub-activity).

The authors introduced the concept of a goal-driven intentional agent - one that has goals that it intends to pursue, "only attempts to perform those actions that are intended and does so without delay." To represent the intentions and decisions of an intentional agent, Blount et al. introduced mental fluents and actions. Two important mental fluents are $\operatorname{status}(\mathbf{m}, \mathbf{k})$ ( $\mathbf{m}$ is in progress if $\mathbf{k} \geq 0$, and not yet started or stopped if $\mathbf{k}=-1$ ) and next_action $(\mathbf{m}, \mathbf{a})$ (the next action to be executed as part of activity $\mathbf{m}$ is a). Mental actions included select(g) and abandon(g) for goals, and $\operatorname{start}(\mathbf{a})$ and stop $(\mathbf{a})$ for activities. The new theory of intentions was encoded in action language $\mathcal{A L}$ (Baral and Gelfond 2000). We denote by new $\mathcal{T} \mathcal{I}$ its ASP translation.

Additionally, Blount et al. developed an agent architecture $\mathcal{A I} \mathcal{A}$ (implemented in CRProlog (Balduccini and Gelfond 2003: Balduccini 2007), an extension of ASP) that adapts the agent loop (Balduccini and Gelfond 2008) to specify the behavior of a goal-driven intentional agent. For instance, while fluent next_action $(\mathbf{m}, \mathbf{a})$ in the theory of intentions indicates the action in activity $\mathbf{m}$ that the agent would normally need to execute next, the agent architecture handles exceptions to this rule. The decision not to execute the next action is made if the activity's goal was already achieved by some other action (Example 2) or was abandoned; or if the current activity needs to be stopped altogether because it no longer has chances of achieving its goal (Example 3).

\subsection{Proposed Methodology}

Our methodology describes how to construct an ASP logic program for each input restaurant narrative based on the information given in the text, a background commonsense knowledge base, theories of intentions, and an adapted and extended version of the $\mathcal{A} \mathcal{I} \mathcal{A}$ architecture. Answer sets of the resulting program correspond to a cautious reader's possible mental models of the narrative, which can be used to demonstrate a deep understanding of the story via question answering. By "deep understanding" we mean awareness of the intentions of characters and of the occurrence of actions that were not explicitly stated in the text but would be assumed by a human reader.

Our goal is to focus on the reasoning component. We thus ignore the natural language processing part, which is a difficult task on its own. We distinguish between the story time line containing strictly the events mentioned in the text and the reasoning time line corresponding to the mental model that the reader constructs. We assume that a wide coverage commonsense knowledge base $(\mathcal{K B})$ written in ASP is available to us and that it contains information about a large number of actions, their effects and preconditions, including actions in the stereotypical activity. How to actually build such a knowledge 
base is a difficult research question, but it is orthogonal to our goal. In practice, in order to be able to evaluate our methodology, we have built a basic knowledge base with core information about restaurants in the spirit of Mueller's work (2007).

According to our methodology, for each input text $t$ we construct a logic program $\Pi(t)$ consisting of an input-dependent part and a pre-defined part common to all texts.

The input-dependent part of $\Pi(t)$ (i.e., the logic form obtained by translating the English text $t$ into ASP facts) consists of facts defining objects mentioned in the text as instances of relevant sorts in the $\mathcal{K B}$ and observations about the values of fluents and the occurrences of actions at different points on the story time line. To record observations about fluents and actions, we use predicates st_obs and st_hpd respectively. By $s_{-}$obs $(\mathbf{f}, \mathbf{v}, \mathbf{s s})$ we mean that fluent $\mathbf{f}$ from the $\mathcal{K} \mathcal{B}$ has value $\mathbf{v}$ at time step ss on the story time line, where $\mathbf{v}$ may be true or $\mathbf{f a l s e})$, and $\operatorname{st} h p d(\mathbf{a}, \mathbf{v}, \mathbf{s s})$ indicates that action $\mathbf{a}$ from the $\mathcal{K B}$ was observed to have occurred if $\mathbf{v}$ is true, or not if $\mathbf{v}$ is false, at time step ss on the story time line. Let us illustrate the logic form obtained for a sample scenario.

Example 4 (Input-dependent part (i.e., logic form) for story in Example 1)

The text in Example 1 is translated into a logic form that includes the following facts:

$$
\begin{array}{ll}
\text { customer(nicole). } & \text { st_hpd(enter(nicole, veg_r }), \text { true, } 0) . \\
\text { restaurant(veg_r }) . & \text { st_hpd( order(nicole, lentil_soup, waitress), true, 1). } \\
\text { food(lentil_soup). } & \text { st_hpd(put(waitress, lentil_soup, t), true, 2). } \\
\text { waitress(waitress). } & \text { st_hpd(eat(nicole, lentil_soup), true, 3). } \\
\text { cook(cook1). } & \text { st_hpd(leave(nicole), true, } 4) .
\end{array}
$$

where enter, order, put, eat, and leave are actions described in $\mathcal{K B}$

The pre-defined part of $\Pi(t)$ consists of:

1. The background commonsense knowledge base $\mathcal{K B}$, which contains information about actions and fluents relevant to the restaurant domain, including axioms about the direct, indirect effects and preconditions of actions. These are encoded in ASP using a standard methodology (Gelfond and Kahl 2014) in which predicates holds(f, i) and $\operatorname{occurs}(\mathbf{a}, \mathbf{i})$ denote the beliefs that fluent $\mathbf{f}$ holds at time step $\mathbf{i}$ and action $\mathbf{a}$ occurs at $\mathbf{i}$ respectively. For example, the two rules below encode one direct effect and one executability condition for action $\operatorname{put}(\mathbf{p}, \mathbf{t}, \mathbf{l})$ - person $\mathbf{p}$ puts thing $\mathbf{t}$ on location $\mathbf{l}$ :

$$
\begin{aligned}
& \neg \operatorname{holds}(\operatorname{holding}(P, T), I+1) \leftarrow \operatorname{occurs}(\operatorname{put}(P, T, L), I), \operatorname{holds}(\operatorname{holding}(P, T), I) \text {. } \\
& \text { impossible }(\operatorname{put}(P, T, L), I) \leftarrow \operatorname{location}(L), \neg \text { holds }(\operatorname{holding}(P, T), I) \text {. }
\end{aligned}
$$

2. A theory (or theories) of intentions.

3. A module encoding the stereotypical activity from the perspective of each actor.

4. A reasoning module, encoding (i) a mapping of time points on the story time line into points on the reasoning time line; and (ii) reasoning components that reflect a reader's reasoning process and expected to allow reasoning about serendipitous achievement of goals, decisions to stop futile activities, and diagnosis.

(i) To encode the mapping of story time steps to reasoning time steps we introduce the predicates story_step and step, respectively, as well as the predicate $\operatorname{map}(\mathbf{s}, \mathbf{i})$ to say that story step $\mathbf{s}$ is mapped into reasoning time step $\mathbf{i}$ :

$$
\begin{aligned}
& 1\{\operatorname{map}(S, I): \operatorname{step}(I)\} 1 \leftarrow \text { story_step }(S) . \\
& \neg \operatorname{map}(S, I) \leftarrow \operatorname{map}\left(S_{1}, I_{1}\right), S<S_{1}, I \geq I_{1}, \text { story_step }(S), \text { step }(I) .
\end{aligned}
$$


Observations about the occurrence of actions and values of fluents, recorded from the text using predicates $s t_{-} h p d$ and st_obs, are translated into observations on the reasoning time line, for which we use the predicates $h p d$ and $o b s$, via rules of the type: $h p d(A, V, I) \leftarrow \operatorname{st} h p d(A, V, S), \operatorname{map}(S, I)$.

Finally, "gaps" on the reasoning time line are prevented by the rules smtg_occurs $(I) \leftarrow$ occurs $(A, I)$.

$\leftarrow$ last_assigned $(I), \operatorname{step}(J), J<I$, not $\operatorname{smtg}$ _occurs $(J)$.

where last_assigned $(\mathbf{i})$ is true if $\mathbf{i}$ is the last time step on the reasoning time line that has a correspondent on the story time line.

(ii) Reasoning components are adapted from $\mathcal{A} \mathcal{I} \mathcal{A}$ and expanded to reflect the reasoning process of an outside observer (the cautious reader) instead of that of an agent thinking about its next action. For instance, $\mathcal{A} \mathcal{I} \mathcal{A}$ rules indicating how an agent should select a new activity to satisfy an active goal if the current activity is deemed futile are replaced by a single rule indicating that the mental action of replanning has occurred: $\operatorname{occurs}(\operatorname{replan}(A g, G), I) \quad \leftarrow \quad$ categ_4_hist $(G, I)$, not futile $(A g, G, I)$, not impossible(replan $(A g, G), I)$.

The cautious reader is not expected to guess what new activity the agent decided to start, unless this is explicitly specified in the text.

5. Default information about the values of fluents in the initial situation (e.g., the restaurant is normally open, dishes listed on the menu are normally available, etc.)

The proposed methodology was tested with good results in previous work: in one instance, only the customer role was modeled as a goal-driven agent using new $\mathcal{T} \mathcal{I}$ while other actors were modeled as simple intentional agents using old $\mathcal{T} \mathcal{I}$ (Zhang and Inclezan 2017); in the other case, all characters were modeled as goal-driven agents using the new $\mathcal{T}$ (Inclezan et al. 2018). However, a couple of important research questions still remain about components 2 and 3 of the pre-defined part:

$R Q_{1}$ If we were interested in answering questions about the goals and intentions of the customer only, what are the trade-offs in terms of coverage and performance between viewing only the customer as a goal-driven intentional agent (i.e., using the new $\mathcal{T} \mathcal{I}$ for the customer only and the old $\mathcal{T} \mathcal{I}$ for all other characters - case denoted by new+old $\mathcal{T} \mathcal{I}$ ) versus viewing all characters as goal-driven agents (i.e., using the new $\mathcal{T} \mathcal{I}$ for all of the characters - case denoted by new $\mathcal{T} \mathcal{I}$-only)?

$R Q_{2}$ How should we structure the representation of the stereotypical activity, from the point of view of each actor, in order to maximize coverage and performance?

We define coverage as the number of different types of scenarios that can be processed correctly. Scenario types include: stories with only one customer versus multiple customers, plus the different scenario types listed in Baral et al.'s (2015) work on theory of intentions (e.g., normal, serendipitous, diagnosis scenarios).

In the next section we present our insights into these two research questions.

\section{Research Questions: Insights}

\subsection{Insight \#1: Two Theories of Intentions}

We start by focusing on research question $R Q_{1}$ and analyze the two cases listed above: new+old $\mathcal{T} \mathcal{I}$ and new $\mathcal{T} \mathcal{I}$-only. The case of viewing all characters as simple intentional 
agents and thus using only old $\mathcal{T} \mathcal{I}$ is not an option. This approach is too limiting and does not allow reasoning correctly about scenarios like the one in Example 2 for reasons similar to those related to the script-based approach.

\subsubsection{Coverage}

The advantage of viewing a character as a goal-driven intentional agent (and using the new $\mathcal{T} \mathcal{I}$ instead of the old $\mathcal{T} \mathcal{I}$ to model the character's activity) is that it allows reasoning about the serendipitous achievement of the character's goals and sub-goals. This means that using the old $\mathcal{T} \mathcal{I}$ instead of new $\mathcal{T} \mathcal{I}$ for secondary characters (e.g., the waiter) leads to scenarios like the one in Example 5 not being processed correctly:

Example 5 (Serendipity for Waiter)

Nicole went to a vegetarian restaurant. She ordered a lentil soup. Nicole was in a hurry, so as soon as the waitress laid the dish on the table, Nicole paid for it in cash and said that she didn't need the bill. (The reader is expected to understand that the waitress did not bring the bill to Nicole.)

For the story in Example 5, an answer set would be produced by the new+old $\mathcal{T} \mathcal{I}$ approach, but it would inaccurately state that the waitress did bring the bill to Nicole. This is a drawback for the new+old $\mathcal{T} \mathcal{I}$ case.

On the other hand, the new+old $\mathcal{T} \mathcal{I}$ case allows reasoning about scenarios involving multiple customers, each ordering a different dish as in Example 6 .

Example 6 (Multiple Customers)

Nicole and Sam went to a vegetarian restaurant. She ordered a lentil soup. He ordered a miso soup. They both enjoyed their soups.

In our formalization of the domain, the waiter either maintains an old $\mathcal{T} \mathcal{I}$ sequence of actions for each customer (case new+old $\mathcal{T} \mathcal{I}$ ) or, alternatively, it maintains one new $\mathcal{T} \mathcal{I}$ activity per customer, with the associated goal of serving and billing the customer (case new $\mathcal{T} \mathcal{I}$-only). However, a waiter cannot maintain multiple new $\mathcal{T} \mathcal{I}$ activities at a time, corresponding to multiple customers, because of a current limitation in Blount et al.'s theory of intentions indicating that an agent can only have one top-level active goal at a time. As a result, applying the $n e w \mathcal{T} \mathcal{I}$-only approach to such scenarios would result in no answer sets. Substantial work on goal selection and prioritization is needed in order to lift this restriction. With the new+old $\mathcal{T} \mathcal{I}$ solution, the secondary role of waiter is modeled as a simple intentional agent who does not maintain goals, but rather follows a sequence of intended actions. As a result, the waiter may entertain multiple sequences of actions at a time.

In terms of the scenarios that we found while working on the restaurant narrative corpus, a higher number of examples involved multiple customers in the style of Example 6 (27.5\%) than those requiring to view secondary characters as goal-driven intentional agents as in Example 5 (15\%).

\subsubsection{Performance}

The new $\mathcal{T} \mathcal{I}$, which is required to reason about goal-driven intentional agents, is much more complex than the $o l d \mathcal{T} \mathcal{I}$. A comparison in terms of different measures can be seen 
in Table 2. This has a substantial impact on the performance of a system implemented according to our methodology, especially on input stories that involve diagnosis, which is a combinatorial search for an explanation.

Table 2. Comparison between old $\mathcal{T} \mathcal{I}$ and new $\mathcal{T} \mathcal{I}$

\begin{tabular}{lcc}
\hline \hline Metric & old $\mathcal{T} \mathcal{I}$ & new $\mathcal{T} \mathcal{I}$ \\
\hline Number of rules & 6 & 111 \\
Number of lines of code & 45 & 453 \\
Number of fluents & 1 & 12 \\
Number of actions & 0 & 2 \\
\hline Minimum number of steps on the reasoning time line & $n$ & $n+2$ \\
for a flat sequence/ activity of length $n$ & & \\
\hline \hline
\end{tabular}

Consider for instance the last metric in Table 2 If activities are represented using a hierarchical structure with sub-activities that have associated sub-goals (which is desired, as we will show in the next subsection), then each sub-activity adds two additional time steps on the reasoning time line: one for the mental action of starting the sub-activity and another one for stopping the sub-activity. This happens even when the sub-activity's goal is serendipitously satisfied by some other agent's actions and none of the physical actions in the sub-activity are performed by the agent (see the output for Example 2 in Section 57. Moreover, no physical actions of the same agent can occur while a mental action is happening, and some restrictions about physical actions of other agents also exist. A larger number of steps on the reasoning time line has an impact on diagnosis problems especially, as shown in Table 3. The reported times are the averages of ten runs on a machine with an Intel(R) Core(TM) i5-4300U CPU 1.9 GHz and 4GB RAM using the CLINGO4.5.4 solvel

Table 3. Performance comparison. (By Max Step we denote the maximum time step on the reasoning time line when an action is considered to have occurred.)

\begin{tabular}{|c|c|c|c|c|c|}
\hline \multirow{2}{*}{ Scenario } & \multicolumn{2}{|c|}{ new + old $\mathcal{T} \mathcal{I}$} & \multicolumn{2}{|c|}{ new $\mathcal{T} \mathcal{I}$-only } & \multirow[b]{2}{*}{ Time increase } \\
\hline & Avg. Time & Max Step & Avg. Time & Max Step & \\
\hline Normal & $1.07 \mathrm{~s}$ & 29 & $1.61 \mathrm{~s}$ & 33 & $50.74 \%$ \\
\hline Serendipity & $1.91 \mathrm{~s}$ & 23 & $2.52 \mathrm{~s}$ & 27 & $32.25 \%$ \\
\hline Futile Activity & $1.03 \mathrm{~s}$ & 8 & $1.34 \mathrm{~s}$ & 9 & $29.89 \%$ \\
\hline Diagnosis 1 (wrong dish) & $2.34 \mathrm{~s}$ & 16 & $3.62 \mathrm{~s}$ & 20 & $54.80 \%$ \\
\hline Diagnosis 2 (wrong bill) & $1.28 \mathrm{~s}$ & 23 & $1.95 \mathrm{~s}$ & 28 & $51.98 \%$ \\
\hline
\end{tabular}

Answer for $R Q_{1}$. Based on this analysis, we conclude that new + old $\mathcal{T} \mathcal{I}$ has an improved

\footnotetext{
${ }^{1}$ https://sourceforge.net/projects/potassco/files/clingo/
} 
performance over new $\mathcal{T} \mathcal{I}$-only and has the potential for a wider coverage as it can handle a larger number of what seem to be recurrent scenarios.

\subsection{Insight \#2: Hierarchical Activity Representation}

To answer research question $R Q_{2}$ about the most suitable structure for the representation of each actor's actions as part of the stereotypical activity, we started from the flat and fixed scripts described in the existing body of literature on narratives about restaurants with table service (e.g., (Schank and Abelson 1977; Mueller 2007)), which we then refined. We considered two main factors that impact decisions about activity structure: (1) in order to be able to reason about serendipitous scenarios, activities must have a hierarchical structure with sub-activities having their own sub-goals; and (2) in order to be able to process scenarios that require diagnosis (e.g., wrong dish / bill) additional parameters may be needed (e.g., one parameter indicating the ordered dish and another one for the actual, possibly wrong, dish brought by the waiter). In what follows, we describe our conclusions related to such decisions, and especially their impact on coverage and performance. We adopt the conclusion from Section 4.1 and assume that the customer's actions are represented as an activity of $n e w \mathcal{T} \mathcal{I}$, while the waiter and cook intend to execute sequences of actions of old $\mathcal{T} \mathcal{I}$.

\subsubsection{Activity Structure and Serendipitous Scenarios}

In our methodology, reasoning about serendipitous scenarios is possible whenever the customer's actions whose purpose is satisfied by someone else's actions are grouped into a sub-activity associated with a goal. For instance, Example 2 can be processed correctly if and only if the customer's activity contains a sub-activity consisting of the paymentrelated actions (request bill and pay bill) and associated with a goal that can be satisfied by another character's actions. When this is the case, the rules in new $\mathcal{T} \mathcal{I}$ indicate that the customer performs the mental action of starting the payment sub-activity, realizes that the goal is already met, and then performs the mental action of stopping the sub-activity, without performing any of the physical actions in it. To increase the coverage of different serendipitous scenarios, we must make sure that we create a hierarchical structure for the customer's activity in which all goals that may be satisfied by other actors' actions are represented and associated with a corresponding sub-goal, thus rendering sub-activities optional. This is the criterion that we employ to divide the customer's activity into subactivities, of course in addition to grouping together the actions that are intuitively part of the same sub-plan (e.g., picking up the menu and putting it back on the table are part of the sub-plan of deciding what to order).

One possibility that would guarantee maximum coverage is to package each action into a sub-activity with a sub-goal as in Activity Theory (Bertelsen and Bødker 2003), and then build other sub-activities from there. However, this would be detrimental in terms of performance. Each new sub-activity that is introduced adds two mental actions (start and stop) that need to be executed by the actor, which translates into two additional time points on the reasoning time line given that no other actions, physical or mental, can be executed by the actor at the same time. As a result, this approach would roughly triple the length of the reasoning time line, as compared to a flat activity, which will negatively 
impact the code that maps story time line steps onto the reasoning time line, as well as scenarios with diagnosis, as shown in the previous section. As an example, consider the structures shown in Table 4 , where $S_{1}$ only introduces one sub-activity compared to $S_{\text {flat }}$. The average time over ten runs for processing a normal scenario using the new+old $\mathcal{T} \mathcal{I}$ approach is $0.57 \mathrm{~s}$ for $S_{\text {flat }}, 0.70 \mathrm{~s}$ for $S_{1}$ (22\% increase), and $1.07 \mathrm{~s}$ for $S_{2}$ ( $87 \%$ increase).

There is an obvious trade-off between coverage and performance that impacts the activity structure we choose. We decided where to draw the line based on the exceptional scenarios in our restaurant corpus that were not hand-crafted. We identified as optimal the activity structure $S_{2}$ shown in Table 4 which includes sub-activities for the customer getting ready to eat (c_subact_r $(C, R, W, F)$ ), customer deciding what to order $\left(c \_s u b a c t \_o(C, F, W)\right)$ and customer paying the bill $\left(c_{-}\right.$subact_p $\left.(C, W)\right)$. Note that $c_{-}$subact_r $(C, R, W, F)$ is optional in a scenario where the wrong dish is brought by the waiter and the customer decides to eat it - at this point the customer drops his initial intention of eating the original dish and starts a new activity of eating the wrong dish, but all the actions up to eating (e.g., sit) become irrelevant and should be made optional.

Table 4. Possible activity structures for the customer role.

\begin{tabular}{|c|c|}
\hline$S_{\text {flat }}$ & $\begin{array}{ll}\text { Name: } & \boldsymbol{c}_{-} \text {act }(\boldsymbol{C}, \boldsymbol{R}, \boldsymbol{W}, \boldsymbol{F}) \\
\text { Plan: } & {[\text { enter }(C, R), \text { lead_to }(W, C, \mathrm{t}), \text { sit }(C), \text { pick_up }(C, \mathrm{~m}, \mathrm{t}), \text { put }(C, \mathrm{~m}, \mathrm{t}),} \\
& \quad \text { order }(C, F, W), \text { eat }(C, F), \text { request }(C, \mathrm{~b}, W), \text { pay }(C, \mathrm{~b}), \text { stand_up }(C), \\
& \text { move }(C, \mathrm{t}, \text { entrance }), \text { leave }(C)] \\
\text { Goal: } & \text { satiated_and_out }(C)\end{array}$ \\
\hline \multirow[t]{2}{*}{$S_{1}$} & 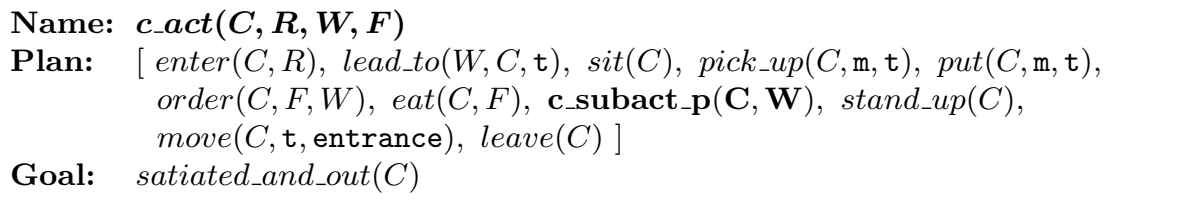 \\
\hline & $\begin{array}{ll}\text { Name: } & \text { c_subact_p }(\mathbf{C}, \mathbf{W}) \\
\text { Plan: } & {[\text { request }(C, \mathrm{~b}, W), \text { pay }(C, \mathrm{~b})]} \\
\text { Goal: } & \text { done_with_payment }(C)\end{array}$ \\
\hline \multirow{4}{*}{$S_{2}$} & 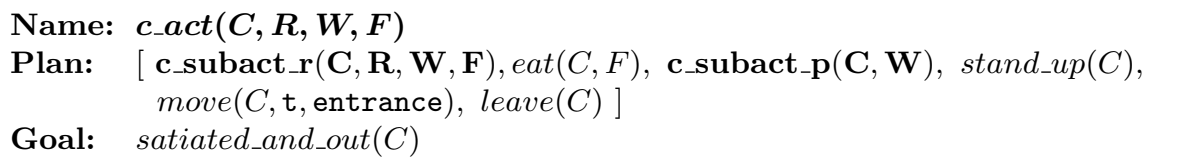 \\
\hline & $\begin{array}{ll}\text { Name: } & \text { c_subact_r }(\mathbf{C}, \mathbf{R}, \mathbf{F}, \mathbf{W}) \\
\text { Plan: } & {\left[\text { enter }(C, R), \text { lead_to }(W, C, \mathbf{t}), \operatorname{sit}(C), \mathbf{c}_{-}{ }_{\text {subact }} \_\mathbf{o}(\mathbf{C}, \mathbf{F}, \mathbf{W})\right]} \\
\text { Goal: } & \text { ready_to_eat }(C)\end{array}$ \\
\hline & $\begin{array}{ll}\text { Name: } & \text { c_subact_o }(\mathbf{C}, \mathbf{F}, \mathbf{W}) \\
\text { Plan: } & {[\text { pick_up }(C, \mathrm{~m}, \mathrm{t}), \operatorname{put}(C, \mathrm{~m}, \mathrm{t}), \operatorname{order}(C, F, W)]} \\
\text { Goal: } & \text { order_transmitted }(C)\end{array}$ \\
\hline & Name: $\mathbf{c}_{-}$subact_p $(\mathbf{C}, \mathbf{W}) \quad$ - as defined in $\boldsymbol{S}_{\mathbf{2}}$ \\
\hline
\end{tabular}




\subsubsection{Activity Parameters and Scenarios Requiring Diagnosis}

We wanted our methodology to be able to encompass exceptional scenarios that require diagnosis (e.g., the waiter brings the wrong dish/bill). We illustrate our analysis and decision process on the waiter role. Recall from Section 4.1 that we decided to model the waiter as a simple intentional agent that follows a sequence of intended actions according to the tenets captured by old $\mathcal{T} \mathcal{I}$.

For each input text, our program generates all possible sequences of actions that the waiter may have intended and determines the actual one based on the events described in the story. For instance, for the story in Example 3 that mentions both a lentil soup and a miso soup, our program assumes that the waitress may be executing a sequence of actions that includes serving either a lentil soup to Nicole or a miso soup. Given that the story indicates that the waitress brought the miso soup, the program will determine that the waitress is executing the latter sequence. We distinguish between one sequence and the other via parameters of the waiter sequence. The dish that is actually served must be one of them as captured by the third parameter of the waiter sequence in (1) below.

In order to account for possible mistakes in communication as well as for careless waiters that do not check the customer's order before serving the dish, we must distinguish between the food order that the waiter understands and the food he serves: $F_{1}$ and $F_{2}$ in the improved version of the waiter sequence shown in (2). Finally, by analyzing the stories in our restaurant corpus, we determined that another recurrent mistake is the wrong bill being brought by the waiter. Thus, we concluded that the waiter sequence must contain an additional parameter representing the bill $B$ that the waiter brings as in (3) and which may not be the customer's actual bill.

$$
\begin{gathered}
w \_s e q(W, C, F) \\
w \_s e q\left(W, C, F_{1}, F_{2}\right) \\
w \_s e q\left(W, C, F_{1}, F_{2}, B\right)
\end{gathered}
$$

The waiter's sequence $w_{-} s e q\left(W, C, F_{1}, F_{2}, B\right)$ can be read as "waiter $\mathrm{W}$ understands that customer $\mathrm{C}$ ordered food $F_{1}$, serves food $F_{2}$ and brings bill $B$ to him/her." It consists of the following actions: $\langle\operatorname{greet}(W, C)$, lead_to $(W, C, \mathrm{t})$, move $(W, \mathrm{t}, \mathrm{kitchen})$, request $\left(W, F_{1}, \mathrm{ck}\right)$ ( $W$ requests to the cook to prepare food $\left.F_{1}\right)$, pick_up $\left(W, F_{2}\right.$, kitchen) ( $W$ picks up food $F_{2}$ from the kitchen), move $(W$, kitchen, $\mathrm{t}), \operatorname{put}\left(W, F_{2}, \mathrm{t}\right)$, move $(W, \mathrm{t}$, counter), pick_up ( $W, B$, counter) ( $W$ picks up bill $B$ from the counter), move ( $W$, counter, $\mathrm{t})$, $\operatorname{put}(W, B, \mathrm{t})\rangle$.

Similarly, we define the cook's intended sequence of actions as $c k_{-} s e q(C k, F, W)$, read as "cook $C k$ understands that he has to prepare food $F$ for waiter $W$ ", where $F$ is not necessarily the food ordered by the customer. A cook's sequence $c k_{-} s e q(C k, F, W)$ consists of a single action: $\langle\operatorname{prepare}(C k, F, W)\rangle$.

Note that for the scenario in Example 3, the program will determine that the customer is executing the activity shown on the first row of Table 5, while the waiter and cook are executing the sequences of actions corresponding to one of the possible cases shown in the same table. Non-matching food items in the customer, waiter, and cook's actions are possible, due to axioms in the $\mathcal{K B}$ that specify that the actions of ordering a food, requesting a food to be prepared by the cook, preparing the food, and picking up a food from the kitchen have non-deterministic effects if a non-agent action (i.e., exogenous 
action) that we call interference occurs simultaneously. For instance, the first axiom below says that an interference occurring at the same time as a customer's action of ordering some food $F$ causes the waiter to understand that the customer is asking for a different food than $F$ :

$$
\begin{array}{r}
1\{\text { holds }(\text { informed }(W, F 1, C), I+1) \text { : other_food }(F 1, F)\} 1 \leftarrow \\
\text { occurs }(\text { order }(C, F, W), I), \text { occurs }(\text { interference, } I) . \\
\text { other_food }\left(F_{1}, F\right) \leftarrow \quad \operatorname{food}(F), \operatorname{food}\left(F_{1}\right), F \neq F_{1} . \\
\text { holds }(\text { informed }(W, F, C), I+1) \leftarrow \operatorname{occurs}(\text { order }(C, F, W), I), \\
\neg \text { occurs }(\text { interference, } I) .
\end{array}
$$

\begin{tabular}{|c|c|}
\hline Customer: & c_act(nicole, veg_r, waitress, lentil_soup) \\
\hline Case 1: & $\begin{array}{l}\text { w_seq(waitress, nicole, lentil_soup, miso_soup, } \mathrm{b}) \\
c k \_s e q(\operatorname{cook} 1, \text { miso_soup, miso_soup) } \\
\text { Explanation: The cook misunderstood the food request made by the waiter. }\end{array}$ \\
\hline Case 2: & $\begin{array}{l}\text { w_seq(waitress, nicole, lentil_soup, miso_soup, } \mathrm{b}) \\
c k \_s e q(\operatorname{cook} 1 \text {, lentil_soup, miso_soup) } \\
\text { Explanation: The cook understood the order correctly but prepared the wrong foo }\end{array}$ \\
\hline Case 3: & $\begin{array}{l}\text { w_seq(waitress, nicole, miso_soup, miso_soup, } \mathrm{b}) \\
c k \_s e q(\text { cook1, miso_soup, miso_soup) } \\
\text { Explanation: The waitress misunderstood the customer's order. }\end{array}$ \\
\hline Case 4: & $\begin{array}{l}\text { w_seq(waitress, nicole, lentil_soup, miso_soup, b) } \\
c k \_s e q(\operatorname{cook1} \text {, lentil_soup, lentil_soup) } \\
\text { Explanation: The waitress picked up the wrong order from the kitchen. }\end{array}$ \\
\hline
\end{tabular}

Table 5. Possible explanations for Example 3.

Answer for $R Q_{2}$. Based on our analysis, we decided to structure the customer's activity as a hierarchical one by introducing sub-activities and sub-goals that would allow us to reason about a large number of serendipitous scenarios (see structure $S_{2}$ in Table 4 . We also added parameters to the waiter and cook's sequences of actions, named $w_{-} s e q\left(W, C, F_{1}, F_{2}, B\right)$ and $c k \_s e q(C k, F, W)$ respectively, to be able to target diagnosis scenarios. We made sure that the $\mathcal{K} \mathcal{B}$ contained axioms about the non-deterministic effects of certain actions when an interference exogenous action occurs simultaneously.

\section{Exemplification of the Refined Methodology}

We employed our answers to research questions $R Q_{1}$ and $R Q_{2}$ to produce a refined methodology for the understanding of restaurant scenarios. In this section, we include the outputs generated by our methodology for three different types of stories and indicate the understanding demonstrated for each of them.

Normal Scenario in Example 1. We use this case as a baseline when explaining the output of exceptional scenarios. The answer set of the program $\Pi(1)$ obtained according 
to our refined methodology contains the occurs $(\mathbf{m a}, \mathbf{i})$ and $\operatorname{intend}(\mathbf{s}, \mathbf{i})$ atoms shown below, where $\mathbf{m a}$ is a mental action, $\mathbf{s}$ is a sequence of actions, and $\mathbf{i}$ is a time point on the reasoning time line:

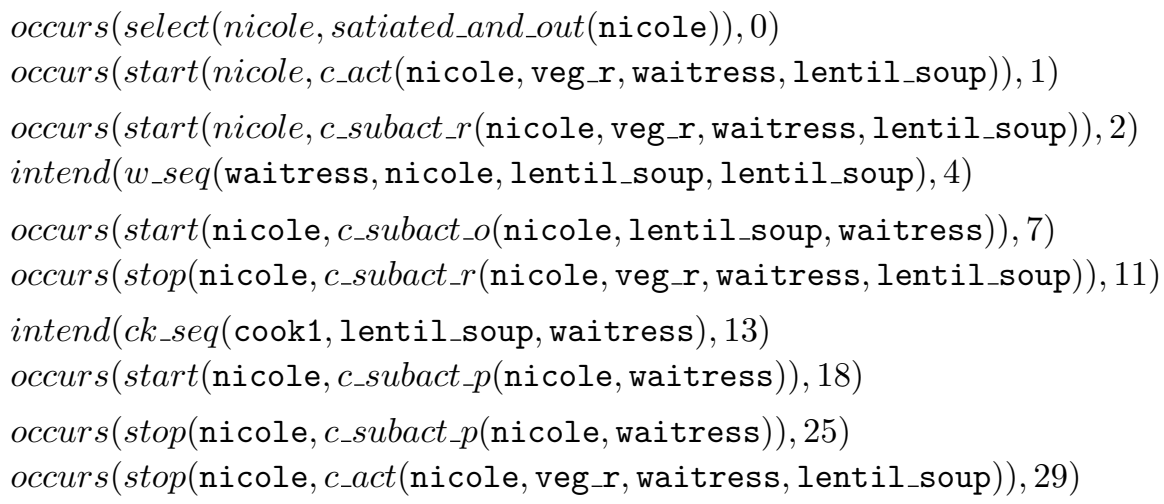

Serendipitous Achievement of Goal in Example 2. The logic form for this scenario is identical to the one for Example 1 shown in Example 4 . except that the two observations about actions taking place at time points 2-4 are replaced by

st_hpd(pay(owner, b), true, 2). st_hpd(put(waitress, lentil_soup, t), true, 3).

where owner is a new instance of sort people. The answer set of $\Pi(2)$ contains similar occurs atoms to $\Pi(1)$ plus one for action pay(owner, b) up to time step 18 when the customer's sub-activity related to the bill payment, $c_{-} s u b a c t \_p$, starts. From then on, it contains the following occurs predicates for mental actions:

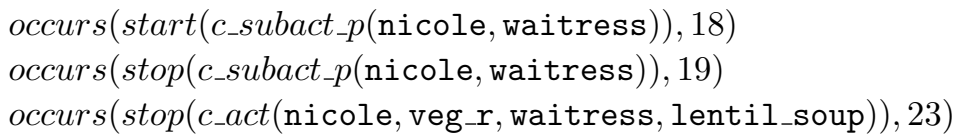

Thus, the reader of this scenario understands that Nicole has stopped $c_{-}$subact_2 immediately after starting it because she realized that its goal is already fulfilled. Based on this answer set, questions about facts not mentioned in the narrative can be answered correctly: Did Nicole pay for the soup? (No); Did Nicole leave the restaurant? (Yes).

Diagnosis in Example 3. The logic form contains the observations:

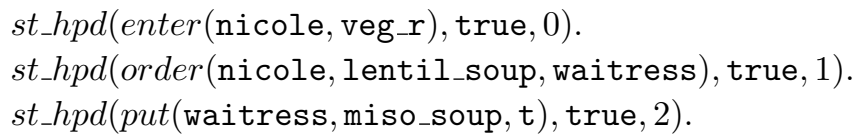

Program $\Pi(3)$ has four answer sets, containing explanations on what may have gone wrong. We illustrate here only one of them, which corresponds to Case 3 in Table 5 the waitress misunderstood the order to be for miso soup and the cook followed her request. This answer set contains the same occurs predicates as $\Pi(1)$ up to time step 9, and considers that the following physical actions occurred at time step 10:

occurs (interference, 10$)$ occurs(order(nicole, lentil_soup, waitress), 10)

while the following sequences of actions were executed by the waitress and cook:

intend( $w \_s e q($ waitress, nicole, miso_soup, miso_soup) $\left.), 4\right)$

intend(c_seq(cook1, miso_soup, waitress $), 13)$ 


\section{Restaurant Narrative Corpus}

Mueller's work (2007) is the most extensive investigation on restaurant stories. For the purpose of training and evaluating his system, Mueller created two corpora: a web corpus containing 800 texts downloaded from the Internet and likely to involve dining in a restaurant, and a Gutenberg corpus obtained by downloading thirty American literature texts from the Project Gutenberg archive ${ }^{2}$ Mueller's corpora are proprietary; attempts to recreate them indicated that this task requires substantial human effort. Due to the lack of available corpora, we assumed the task of creating a benchmark collection of restaurant stories, which resulted in a corpus we call restaurant-1.0.3 We believe that making this corpus available to the research community is important in facilitating research and allowing for comparisons between systems. The collection can be useful to researchers in a variety of fields including the NLP community, or those bridging between the NLP and KRR communities.

When deciding what stories to include in our corpus, we strove to satisfy the following desired properties, adopted from previous work on ASP benchmark set selection ( et al. 2013):

$\left(P_{1}\right)$ Broad selection, i.e., using a variety of sources for the excerpts;

$\left(P_{2}\right)$ Fair selection, i.e., one source should not dominate other sources in terms of representation in the corpus;

$\left(P_{3}\right)$ Adapted hardness, i.e., stories and questions should not be too easy nor too hard from the KRR point of view, as well as with respect to the NLP task of producing logic forms from natural language texts, which we plan to automate in the future;

$\left(P_{4}\right)$ Free of duplicates, reproducible, and publicly available (duplication of excerpts was an issue for Mueller's web corpus).

To satisfy desired property $P_{1}$, we selected excerpts form a variety of sources for inclusion in the restaurant-1.0 corpus: Youtube videos about restaurant scenarios intended for English as a Second Language (ESL) learners, texts available via Google Books, Project Gutenberg texts, stories from Mueller's paper (2007), and hand-crafted scenarios. Table 6 shows the distribution of corpus excerpts. There is a somewhat balanced representation of sources, if we ignore excerpts retrieved from Mueller's paper and Project Gutenberg, which shows at least a moderate satisfaction of desired property $P_{2}$.

To address property $P_{3}$, we made sure not to include stories that explicitly mentioned less than two restaurant-related events (so that the excerpts would not be too hard) nor stories that omitted less than three such events (not too easy). Given that we focused on the KRR task, we wanted to make sure that the excerpts in the corpus could be handled with moderate to high accuracy by existing NLP tools like LTH (Johansson and Nugues 2007b; Johansson and Nugues 2007a) or CORENLP (Manning et al. 2014), while still sounding natural to a native speaker of English. To do so, we adapted the original stories by transforming dialogs into narratives, extracting only the sentences directly related to the restaurant scenario, and simplifying some of them, while not removing co-references for instance. More details are provided below when we describe the process for each source of excerpts.

2 http://www.gutenberg.org/

3 'The corpus is available at https://ceclnx01.cec.miamioh.edu/ inclezd/Restaurant1.0/ 
Table 6. Distribution of excerpts in the restaurant-1.0 corpus

\begin{tabular}{|c|c|c|c|c|c|}
\hline \multirow[t]{2}{*}{ Source } & \multicolumn{4}{|c|}{ Number of excerpts } & \multirow[t]{2}{*}{ Percentage } \\
\hline & Normal & Exception & Variation & Total & \\
\hline Youtube videos & 6 & 6 & 0 & 12 & $30.0 \%$ \\
\hline Google Books & 4 & 3 & 5 & 12 & $30.0 \%$ \\
\hline Project Gutenberg & 0 & 2 & 0 & 2 & $5.0 \%$ \\
\hline Mueller $(2007)$ & 1 & 0 & 0 & 1 & $2.5 \%$ \\
\hline Hand-crafted & 2 & 11 & 0 & 13 & $32.5 \%$ \\
\hline Total & 13 & 22 & 5 & 40 & $100.0 \%$ \\
\hline
\end{tabular}

To satisfy desired property $P_{4}$, we made the corpus publicly available. For each story in the corpus, we recorded the adapted excerpt (what Mueller calls "condensed narrative"), the source, the scenario type (normal/exception/variation), and our logic form encoding in ASP according to the description in Section 3.3 . We believe that including the manually produced logic forms in the corpus represents a useful resource for NLP research. Each story was assigned a unique ID. The raw data can be downloaded as an XML file and can be viewed online in the format illustrated in Figure 1. Given the manageable size of the corpus, we were easily able to ensure that no duplicates are present. Moreover, we made sure that our corpus did not include stories that were roughly equivalent at the level of the ASP logic form (e.g., we discarded stories with the same explicitly mentioned events and same number of customers, but with different customer names). To make sure that the corpus is largely reproducible, we present next the process that we used for adapting stories from the different sources into corpus excerpts.

Scenario \#27:
Nicole went to a vegetarian restaurant. She ordered lentil soup. The waitress set the soup in
the middle of the table. Nicole enjoyed the soup. She left the restaurant.
- Mueller, E.T. 2007. Modelling space and time in narratives about restaurants. Literary and Linguistic Computing
22, 1,67-84.
Logic Form:
restaurant (veg_r). customer(nicole). food(lentil_soup). waiter(waitress).
story_step(0..4). st_hpd(enter(nicole, veg_r), true, 0). st_hpd(order
(nicole, lentil_soup, waitress), true, 1). st_hpd(put_down(waitress,
lentil_soup, t), true, 2). st_hpd(eat(nicole, lentil_soup), true, 3).
st_hpd(leave(nicole), true, 4).

Fig. 1. Online display for the restaurant-1.0 corpus

Youtube videos. We searched www.youtube.com using the query "ESL restaurant." We considered the first 20 results. Many search results were videos of instructors or actors acting out a simple, stereotypical restaurant scenario. The videos were meant to help non-native English speakers learn the restaurant-related vocabulary and explain the process of dining at a restaurant with table service. These videos were intentionally simple and thorough. We discarded videos that were not about restaurants with table 
service, or videos that did not tell a story, and ended up with twelve videos. A native speaker of English translated each video into a narrative that captured not only the restaurant-related dialog, but also the actions performed by the actors.

Google Books. We searched Google Books using the Boolean query indicated by Mueller (2007): "the menu" AND ("the waiter brought" OR "the waiter placed" OR "the waiter set" OR "the waiter put" OR "the waiter poured"). For each identified book, we made sure that the whole text related to the restaurant episode was available in Google Books. As a result, we obtained twelve book excerpts. From each excerpt, we removed sentences not related to what happened in the restaurant or conversations between characters on unrelated topics. Finally, we transformed remaining dialogs into narratives.

Project Gutenberg. Substantially more time and effort were needed to retrieve restaurant examples from this resource, in comparison with the previous two sources. We downloaded 20 texts and applied the process outlined for Google Books above. Unfortunately, many of the texts returned by the search were not useful, as they mentioned explicitly only two or less restaurant-related actions. Only two stories in the corpus are obtained from Project Gutenberg.

Mueller (2007). We included in the corpus the only sample story included in Mueller's paper (see Example 1).

Hand-crafted. We considered the different types of scenarios listed in Blount et al. (2015) and determined for each of them whether a similar case could be captured by a restaurant story. It is important to note that the scenarios conceived by Blount et al. consider the perspective of a single agent thinking about its own intentions and making decisions about what action to execute next. Instead, in a restaurant story, the reader learns about the actions performed by the several actors that are involved, but the reader is merely an observer who does not make decisions about actions. We can also assume that the reader is cautious and thus does not jump to conclusions that are not supported by the story. For instance, when learning that a customer was brought a wrong dish, the cautious reader will not assume that the customer complained nor that he decided to eat the wrong dish, unless such information is explicitly stated in the text. We believe the subset of restaurant-1.0 consisting of the thirteen hand-crafted excerpts is particularly useful to the research community, as it covers a considerable variety of exceptional cases.

Inspired by Mueller's idea of automatically generating questions to test the understanding of restaurant narratives, we expanded our corpus with an ASP module, also available online, that can generate a number of queries for each excerpt. The module produces questions of the forms described in (Inclezan et al. 2018):

- query_yes_no $(A)$ - Did action $A$ occur?

- query_when $(A)$ - When did action $A$ occur?

- query_where $(P, A)$ - Where was person $P$ when action $A$ happened?

- query_who $(A)$ - Who performed action $A$ ?

- query_who_whom $(A)$ - Who performed action $A$ and to whom?

- query_what $(F, A)$ - What was the value of fluent $F$ when action $A$ happened?

- query_goal $(P, A)$ - What goal was $P$ trying to achieve when action $A$ happened?

- query_intended $(P, A)$ - What was $P$ 's activity/sequence of intended actions when action $A$ happened? 
where $A$ is a physical action. Queries are generated based on the $\mathcal{K} \mathcal{B}$ vocabulary and the entity names identified in the logic form associated with an input text, using rules like:

$n\left\{q u e r y\left(y e s \_n o(A)\right):\right.$ physical_action $(A)$, not explicit_in_story $\left.(A)\right\} \mathrm{m}$.

explicit_in_story $(A) \leftarrow$ st_hpd $(A, B, S)$.

Note that only questions about information not explicitly stated in the text are generated. The user can set the number of questions of each type that are to be produced by setting the constants $n$ and $m$ to desired values.

\section{Conclusions and Future Work}

In this paper, we have extended our previous work on modeling restaurant stories using ASP and theories of intentions. We have shown that our approach is suitable for reasoning about stories containing exceptions to the normal unfolding of a restaurant episode, which could not be processed by previous script-based approaches. We have addressed two research questions geared towards refining the methodology - choice of theory of intentions and representation of activities - in order to increase coverage and performance. Additionally, we have presented a corpus of restaurant stories that is publicly available. This will be a useful resource for researchers in the KRR community working on stereotypical human activities, but also to researchers in the NLP field.

In the future, we plan to expand our corpus with new sources, new excerpts, and new record fields to describe the excerpts (e.g., original book fragment/dialog). We are also working on evaluating the applicability of our methodology to other stereotypical activities (e.g., doctor visit) and creating a similar story corpus for other activity domains.

\section{Acknowledgments}

We would like to thank Zengzhi Jiang, Keya Patel, and Marcello Balduccini for their help in retrieving excerpts from Google Books and Project Gutenberg.

\section{References}

Balduccini, M. 2007. CR-MODELS: An inference engine for CR-Prolog. In Proceedings of LPNMR 2007, C. Baral, G. Brewka, and J. S. Schlipf, Eds. LNCS, vol. 4483. Springer, 18-30.

Balduccini, M. And Gelfond, M. 2003. Logic Programs with Consistency-Restoring Rules. In Proceedings of Commonsense-03. AAAI Press, 9-18.

Balduccini, M. And Gelfond, M. 2008. The AAA architecture: An overview. In Architectures for Intelligent Theory-Based Agents, Papers from the 2008 AAAI Spring Symposium, 2008. AAAI Press, 1-6.

Baral, C. And Gelfond, M. 2000. Reasoning Agents in Dynamic Domains. Kluwer Academic Publishers, Norwell, MA, 257-279.

Baral, C. And Gelfond, M. 2005. Reasoning about Intended Actions. In Proceedings of AAAI-05. AAAI Press, 689-694.

Barr, A. And Feigenbaum, E. 1981. The Handbook of Artificial Intelligence. Vol. 1. William Kaufman Inc., Los Altos, CA.

Bertelsen, O. W. And BøDKer, S. 2003. Activity Theory. HCI models, theories, and frameworks: Toward a multidisciplinary science. Morgan Kaufmann, San Franciso, CA, 291-324.

Blount, J. 2013. An Architecture for Intentional Agents. Ph.D. thesis, Texas Tech University, Lubbock, TX, USA. 
Blount, J., Gelfond, M., And Balduccini, M. 2015. A theory of intentions for intelligent agents. In Proceedings of LPNMR 2015, F. Calimeri, G. Ianni, and M. Truszczynski, Eds. LNCS, vol. 9345. Springer, 134-142.

Bratman, M. 1987. Intentions, Plans, and Practical Reason. Harvard University Press, Cambridge, MA.

Chambers, N. And Jurafsky, D. 2008. Unsupervised learning of narrative event chains. In Proceedings of ACL-08: HLT. 789-797.

Craik, K. J. W. 1943. The Nature of Explanation. Cambridge University Press, Cambridge.

Gabaldon, A. 2009. Activity recognition with intended actions. In Proceedings of IJCAI 2009, C. Boutilier, Ed. 1696-1701.

Gelfond, M. And Kahl, Y. 2014. Knowledge Representation, Reasoning, and the Design of Intelligent Agents. Cambridge University Press.

Gordon, A. S., CaO, Q., And Swanson, R. 2007. Automated story capture from internet weblogs. In Proceedings of the 4th International Conference on Knowledge Capture. K-CAP '07. ACM, New York, NY, USA, 167-168.

Gupta, R. And Kochenderfer, M. J. 2004. Common sense data acquisition for indoor mobile robots. In Proceedings of the 19th National Conference on Artifical Intelligence. AAAI'04. AAAI Press, 605-610.

Hoos, H. H., Kaufmann, B., Schaub, T., And Schneider, M. 2013. Robust benchmark set selection for boolean constraint solvers. In 7th International Conference on Learning and Intelligent Optimization (LION-13) - Revised Selected Papers. 138-152.

Inclezan, D. And Gelfond, M. 2011. Representing Biological Processes in Modular Action Language ALM. In Proceedings of Commonsense 2011. 49-55.

InClezAn, D., Zhang, Q., BAlduccini, M., AND IsRANEy, A. 2017. Understanding restaurant stories using an ASP theory of intentions (Extended abstract). In Technical Communications of the 33rd International Conference on Logic Programming (ICLP-TC 2017). OASIcs.

Inclezan, D., Zhang, Q., Balduccini, M., And Israney, A. 2018. An ASP methodology for understanding narratives about stereotypical activities. Theory and Practice of Logic Programming 18, 3-4, 535-552.

Johansson, R. And Nugues, P. 2007a. Language Technology at LTH. http://nlp.cs.1th.se/ [Accessed: 2018].

Johansson, R. And Nugues, P. 2007b. Lth: Semantic structure extraction using nonprojective dependency trees. In Proceedings of the Fourth International Workshop on Semantic Evaluations (SemEval-2007). Association for Computational Linguistics, Prague, Czech Republic, 227-230.

Johnson-LAird, P. N. 1983. Mental Models: Toward a Cognitive Science of Language, Inference, and Consciousness. Harvard University Press, Cambridge, MA.

Manning, C. D., Surdeanu, M., Bauer, J., Finkel, J., Bethard, S. J., And McClosky, D. 2014. Stanford CoreNLP a suite of core NLP tools . http://stanfordnlp.github.io/ CorenLP/ [Accessed: 2018].

Manshadi, M., Swanson, R., And Gordon, A. S. 2008. Learning a Probabilistic Model of Event Sequences From Internet Weblog Stories. In 21st Conference of the Florida AI Society (FLAIRS), Applied Natural Language Processing Track. Coconut Grove, FL.

Modi, A., Anikina, T., Ostermann, S., And Pinkal, M. 2017. InScript: Narrative texts annotated with script information. CoRR abs/1703.05260.

Mueller, E. T. 2004. Understanding script-based stories using commonsense reasoning. Cognitive Systems Research 5, 4, 307-340.

Mueller, E. T. 2007. Modelling space and time in narratives about restaurants. Literary and Linguistic Computing 22, 1, 67-84.

NG, H. T. And Mooney, R. J. 1992. Abductive plan recognition and diagnosis: A comprehensive empirical evaluation. In Proceedings of the 3rd International Conference on Principles 
of Knowledge Representation and Reasoning (KR'92). Cambridge, MA, October 25-29, 1992. 499-508.

Regneri, M., Koller, A., And Pinkal, M. 2010. Learning script knowledge with web experiments. In Proceedings of the 48th Annual Meeting of the Association for Computational Linguistics. ACL '10. Association for Computational Linguistics, Stroudsburg, PA, USA, 979988.

Schank, R. C. And Abelson, R. P. 1977. Scripts, Plans, Goals, and Understanding: An Inquiry into Human Knowledge Structures. Lawrence Erlbaum.

Shanahan, M. 1997. Solving the Frame Problem. MIT Press.

Singh, P., Lin, T., Mueller, E. T., Lim, G., Perkins, T., and Zhu, W. L. 2002. Open mind common sense: Knowledge acquisition from the general public. In On the Move to Meaningful Internet Systems, 2002 - DOA/CoopIS/ODBASE 2002. Springer-Verlag, London, UK, 1223-1237.

Smith, D. And Arnold, K. C. 2009. Learning hierarchical plans by reading simple english narratives. In Proceedings of the Commonsense Workshop at IUI-09.

VAn Dijk, T. A. And Kintsch, W. 1983. Strategies of Discourse Comprehension. Academic Press, Orlando, FL.

Zhang, Q. AND InClezan, D. 2017. An application of ASP theories of intentions to understanding restaurant scenarios. In Proceedings of PAoASP'17. 